\title{
Chapter 10. The Human Respiratory Microbiome: Implications and Impact
}

\section{Alicia B Mitchell and Allan R Glanville}

\section{Abstract}

Once considered a sterile site below the larynx, the tracheobronchial tree and parenchyma of the lungs are now known to harbor a rich diversity of microbial species including bacteria, viruses, fungi and archaea. Many of these organisms, particularly the viruses which comprise the human respiratory virome have not been identified, so their true role is unknown. It seems logical to conclude that a "healthy" respiratory microbiome exists which may be modified in disease states and perhaps by therapies such as antibiotics, antifungals and antiviral treatments. It is likely that there is a critical relationship or equilibrium between components of the microbiome until such time as perturbations occur which lead to a state of dysbiosis or an "unhealthy" microbiome. The act of lung transplantation provides an extreme change to an individual's respiratory microbiome as, in effect, the donor respiratory microbiome is transplanted into the recipient. The mandatory ex vivo period of the donor lungs appears to be associated with blooms of resident viral species in particular. Subsequently, allograft injury, rejection and immune suppressive therapy all combine to create periods of dysbiosis which when combined with transient infections such as community acquired respiratory viruses may facilitate the development of chronic allograft dysfunction in predisposed individuals. As our understanding of the respiratory microbiome is rapidly expanding, based on the use of new generation sequencing tools in particular, it is to be hoped that insights gained into the subtle relationship between the microbiome and the lung allograft will facilitate improved outcomes by directing novel therapeutic endeavors. 


\section{Introduction}

The microbiome consists of all micro-organisms and their products that occupy surfaces within the human body. Each major compartment of the human body appears to have a unique microbiome with species which are specific for that environment. The microbiome encompasses bacteria, fungi, viruses (including bacteriophages) and archaea. These microorganisms are an integral part of the functional human unit. The human body hosts more than a trillion microbial cells and microbiome-associated genes outnumber human-coded genes 100-fold. ${ }^{1}$ Humans and microbes have co-evolved over millions of years, and subsequently, the human immune system and the microbiome demonstrate complex interactions. The development of the microbiome is integral in shaping the immune response, while the immune system is required in order to maintain this large, and highly diverse set of microbes. Thus, a symbiotic interface has been established within the human body. ${ }^{2}$

The advent of culture-independent new technologies such as highly-parallel DNA sequencing facilitated the beginning of the Human Microbiome (HM) Project, which signaled a phase shift from investigating single organisms in isolation, to investigating the whole microbial community. The HM project aimed to characterize the human microbiota and to analyze the role of micro-organisms in health and disease. The focus was on the bacteria found in five main body sites: oropharynx, skin, vagina, gut and nasal cavity. During the HM project, bacterial reference genomes were determined and an open-access database was established to allow collaborative efforts from laboratories worldwide. ${ }^{3,4}$ Collaboration between numerous laboratories has allowed the characterization of commensal bacterial species within a range of body systems. After initially concentrating on developing a reference set composed of a range of normal individuals, the role of microbial imbalance or dysbiosis in different disease states has been investigated. ${ }^{5}$ 
Many modern molecular techniques exploit the 16S ribosomal RNA (rRNA) gene, a small and highly conserved locus contained within all bacterial genomes that allows easy identification of bacterial sequences, and a target for amplification and sequencing. ${ }^{6}$ Once sequenced, these can be referenced against the open-access databases to give genus and species level information. The next-generation sequencing technologies such as the 454 pyrosequencing and Illumina platforms allow whole communities to be characterised simultaneously and for a greatly reduced cost compared to previous sequencing technologies. ${ }^{7}$ The conserved $16 \mathrm{~S}$ rRNA region is largely responsible for the large body of research now available focusing on bacteria, in comparison to the limited data regarding viruses and fungi present within human systems.

Prior to the HM Project, culture-based techniques were used to investigate bacterial presence in the human body, which led to the assumption that the respiratory tract was a sterile site. Accordingly, the lungs were originally omitted from the list of priority sites for the HM project. ${ }^{8}$ This appeared to disregard prior evidence from the 1970's and 80's suggesting that the lungs contained bacteria aspirated from the upper respiratory tract, identified using radiotracers in a group of healthy individuals..$^{9,10}$ Subsequent to the initial efforts in mapping the human microbiome, research studies began to emerge that demonstrated that the lower respiratory tract is home to a diverse range of bacterial species with variations between health and disease states. ${ }^{11}$ In late 2009, the Lung HIV Microbiome Project was established with the aim "To characterize the microbiome of the lung and respiratory tract, and enhance understanding of the role of the lung microbiome in preserving health or causing disease and in the divergent effects observed in HIV-infected versus uninfected individuals."12 Since then, numerous studies have characterised aspects of the bacterial microbiota in a range of acute and chronic respiratory conditions.

The human microbiome is intricately involved in a range of normal and pathological processes during development, and throughout life. Traditionally, it was assumed that the microbiome 
was established at birth, and its composition was dependent on route of delivery. The fetal gut microbiome demonstrates diverse communities within the first week of life, however a more mature equilibrium is not reached until approximately 3 years of age $\mathrm{e}^{13}$. New published evidence indicates the presence of bacterial DNA in amniotic fluid and placental specimens, raising the possibility of microbiome exposure prior to birth. ${ }^{14,15}$ There are differences in the neonatal gut microbiome between full-term and pre-term infants. The major phyla in pre-term infants is Firmicutes compared with a dominance of Actinobacteria in full-term infants, ${ }^{16}$ which further suggests that microbiome seeding may occur during pregnancy and vary with gestational age. Additionally, breastfeeding in the early neonatal period appears to have significant effects on gut microbiota development. In a group of breastfed infants, virulence genes were increased within the gut microbiome, which correlated with up-regulation of immunity-related genes. Early exposure from the mother, both while in utero and in the first few weeks of life, plays an important role in the development of the microbiota in multiple organ systems.

Establishment of the microbiome is crucial in immune development. The effect of early exposure to allergens was evaluated in a murine model, where exposure to house dust mite (HDM) in the early neonatal period lead to increased airway eosinophilia and airway hyperresponsiveness. After establishment of lung microbiota and shifts from Firmicutes and Gammaproteobacteria towards a Bacteriodes predominance during the first two weeks after birth, a decreased response to aeroallergens was observed. An emergence of a $T_{\text {reg }}$ subset which required interaction with programmed death ligand 1 (PD-L1) appeared to be associated with this decreased allergen response. ${ }^{17}$ This demonstrates the importance of airway microbiota establishment in the development of atopic responses and possibly the later development of asthma. During childhood, diet, genetics and environmental exposures affect development of the microbiome, all of which play an important role in immune system maturation. Specifically, the oral microbiota, which in turn influences the lung microbiota, has 
been shown to be influenced by external factors in the first 5 years of life, including breastfeeding, day care attendance and use of antibiotics. ${ }^{18,19}$

\section{The Human Respiratory Microbiome}

The respiratory microbiome includes all airway and lung-tissue associated microbes, and is more specifically defined as the lower respiratory tract beneath the larynx. Above this, the oropharyngeal and nasal-associated microbiota are separate to the lower respiratory tract microbiome and have received more attention previously due to ease of access.

The majority of studies investigating the microbiome have focused on the gut due to the relatively large biomass present and the ease of sampling. Conversely, there are many challenges when attempting to characterize the microbiome of the respiratory tract. In particular, there are difficulties in obtaining samples from the distal airways due to the risk of upper airway contamination. The dominant methodologies employed in sampling the lower respiratory tract include bronchoalveolar lavage (BAL) obtained via bronchoscopy, and spontaneously expectorated or induced sputum. Sputum presents a less invasive method of sampling the lower respiratory tract but has an increased risk of oropharyngeal contamination due to passing directly through the upper respiratory tract. However, when used as a sampling technique in diseases such as cystic fibrosis (CF), bronchiectasis and chronic obstructive pulmonary disease (COPD), the biological signals detected have been significantly and meaningfully associated with multiple measures including severity of illness, airway inflammation, antibiotic use and risk of subsequent exacerbations. ${ }^{20-25} \mathrm{~A}$ further study found that expectorated sputum samples accurately represented the dominant microbes in the relatively homogenous respiratory tract of individuals with end-stage CF who had undergone lung transplantation, however sputum samples overrepresented the diversity and representation of atypical bacterial species. ${ }^{26}$ Bronchoscopy is a more invasive option, which 
may have a lower rate of upper respiratory tract contamination. The bronchoscope is passed through either the oral or nasal cavity to sample the lungs, but once inserted, lavage is collected directly from distal airways without direct passage through the upper respiratory tract. A recent study ${ }^{27}$ using serial $B A L$ analysis, demonstrated that sampling the lungs via bronchoscopy was not significantly confounded by the oral microbiome, consistent with previous serial bronchoscopy studies. ${ }^{28}$ The evidence from these studies supports the understanding that minimal contamination from the upper respiratory tract is present in lower respiratory tract samples when utilising bronchoscopic techniques.

Sampling of the lower respiratory tract and lung tissues has indicated that there are approximately 10-100 bacterial cells per 1000 human cells within the lungs, ${ }^{29}$ a greatly reduced biomass compared with other colonised body compartments. This may be due to lower levels of nutrient sources in the lungs supporting microbiota growth when compared with the gastrointestinal tract. Variable physiological conditions are present within the lungs which may also affect bacterial presence. These factors include $\mathrm{pH}$, relative blood perfusion, relative alveolar ventilation, temperature, oxygen concentration, epithelial cell structure, deposition of inhaled particles, and number of inflammatory cells. ${ }^{30-32}$ These local selective pressures appear to play less of a role in healthy subjects. The greatest impact is seen in severe cases of chronic respiratory conditions where the lung microenvironment becomes maladapted due to remodelling of the airways and extra-cellular matrix. ${ }^{33,34}$

Additionally, lung-associated biomass appears to be significantly lower than the upper respiratory tract biomass. Total bacterial signal level as measured by qPCR for 16S rRNAencoding genes in DNA, showed that the signal level was 100-1000 fold lower in BAL samples when compared with an oral wash. ${ }^{27} \mathrm{~A}$ similar pattern was also observed with species richness, with a higher bacterial species richness in the oral cavity compared with both the lungs and the nasal cavity. This low concentration of microbiota within the lungs may be associated with the early understanding of lung sterility, but even after clear demonstration of 
a resident lung microbiota using sequencing based approaches, the low biomass still presents further challenges in characterizing the burden and diversity of microbial species contained within the lower respiratory tract.

Culture-based protocols characteristic of clinical microbiology laboratories are optimised to detect acute infections with respiratory pathogens in individuals and to distinguish their absence. They rely on sufficient microbial load and specific growth conditions to encourage growth of these bacterial species. ${ }^{35}$ These conditions selectively disadvantage the growth of anaerobes and bacteria which do not optimally flourish at $37^{\circ} \mathrm{C}$, encompassing the majority of

bacterial species which have now been determined to compose the healthy lung microbiota. Many of the species present within the healthy lung determined by culture-independent techniques, have now been grown in culture under modified conditions. The first study to utilize these culture-independent sequencing methodologies to characterize the microbiota in the lungs of healthy subjects showed that the lungs contain a distinct and diverse microbiome. Furthermore, the bacterial composition in the lungs of healthy controls was compared with asthmatic subjects, demonstrating early evidence of variations in the lung microbiota in patients with chronic respiratory diseases, specifically the relative enrichment of Proteobacteria. $^{36}$

Using culture independent techniques, it has since been determined that the most common bacterial phyla in the lower respiratory tract include Bacteroidetes, Firmicutes, and Proteobacteria. The most prominent genera present in healthy controls are Prevotella, Veillonella, and Streptococcus. Studies comparing concurrently collected upper and lower respiratory tract samples show that the microbial communities are distinct from each other, but there are similarities that prevail. This suggests that the traditional understanding of the respiratory tract as comprised of discrete, independent compartments is likely outdated. A more accurate representation of the respiratory tract is a single continuous, internally 
heterogeneous ecosystem from the anterior nares through to the alveoli. ${ }^{27}$ Spatial heterogeneity of the microbial topography within this system occurs within different areas but there is constant movement of species between these areas. ${ }^{11}$

This clear spatial heterogeneity within the different lobes of the lung ${ }^{37}$, and at different levels of the respiratory tract has been observed in chronic respiratory conditions. The turbulent act of coughing may play a role in homogenizing the microbial species within the lumen of the airways, thus decreasing spatial heterogeneity in healthy subjects. ${ }^{28,37}$ In disease states such as CF, where thick, sticky secretions prevent effective clearance of microbes through mucociliary and coughing mechanisms, spatial differences in microbes may be more commonly observed ${ }^{38}$. Lung disease alters the population dynamics and the lung terrain ${ }^{37}$, which translate into unique environmental conditions in each disease state which facilitate changes to the local microbial communities.

The microbiota of the lungs is a balance between immigration of species, likely from microaspiration and direct movement into the lungs, elimination due to mucociliary mechanisms, cough and the innate and adaptive immune responses and selective pressures present within the lungs themselves. Previous studies have shown that microbiota from the oral cavity are constantly being introduced into the lower respiratory tract, often during sleep as a result of microaspiration ${ }^{9,10}$ and likely due to the direct mucosal extension between the oral cavity and the lungs. It has also been suggested that microaerosols generated in the oral cavity may be inhaled into the lungs and further contribute to the composition of the lung microbiome. The bacterial communities within healthy lungs demonstrate significant overlap with the bacterial species found in the oral cavity but not the nasal cavity. However, the relative abundance of bacterial phyla in healthy lungs appears to be significantly different from the oral bacterial community. Selective elimination of Prevotella species was observed in the lung, ${ }^{27}$ providing further evidence for the influence of selective pressures within the lower respiratory tract in shaping the microbiome composition. 
Elimination mechanisms are involved in clearance of microbial species from the respiratory tract. Mucociliary clearance and cough allow organisms to be mechanically removed from the lungs. Microbes are trapped within the mucus secreted from goblet cells and through the constant beating of cilia or coughing are moved up and out of the lower respiratory tract into the pharynx where they are either expectorated or swallowed. Host inflammatory cells and cytokines are involved in the immune response in the lungs leading to the clearance of potential pathogens. The composition of effector cells present within the airway appears to be associated with features of the microbiota. ${ }^{28}$ Therefore, a constant flux in the lung microbiota from introduction of oral microbes and elimination of species present within the lungs as well as selective pressures induced by the lung microenvironment, create a 'steady state' during health (Figure 1). ${ }^{27}$

\section{The Gut-Lung Axis}

The gut microbiome is modified in response to respiratory illness and chronic conditions while the lung microbiome also appears to be influenced by the gut microbiome. This complex interplay between these two body systems and the microbiomes contained within them is dubbed the 'gut-lung axis' and the vital cross-talk between their mucosal surfaces may have important implications for both health and disease. A study in children with CF demonstrates that the lung and gut microbiota likely develop simultaneously after birth. Furthermore, concordant fluctuations in bacterial species at both sites have been observed. ${ }^{39}$ Dietary intake also appears to influence changes in the lung microbiome, in addition to the gut. ${ }^{39,40}$ As mentioned earlier, the early development of the gut microbiota is important in the development of immune responses. The absence of a normal gut microbiota increases susceptibility to lung infection ${ }^{41}$, providing further evidence that the intestinal microbiome may influence the development of a dysbiotic state in the lung microbiome. 
Dysbiosis in both the airway and intestinal microbiota has been observed in the presence of a range of respiratory diseases. ${ }^{42-44}$ Additionally, the course of some respiratory diseases has been shown to be modified by shifts in intestinal microbiota, such as the development of asthma and other atopic conditions. ${ }^{42,44}$ The hygiene hypothesis stipulates that reduction in environmental exposures, use of antibiotics and dietary changes can lead to disruptions of the gut microbiota, which in turn, leads to a decrease in immune tolerance. This resultant decrease in the Th1 immune response leads to a shift towards the propensity for allergic airway changes. ${ }^{45}$ In a murine model, early exposure to antibiotics caused a reduction in microbial load and diversity, which was strongly associated with the later development of allergic airway inflammation in adults who were exposed to aeroallergens. ${ }^{46}$ Conversely, the oral administration of probiotics including Lactobacillus species to children with CF led to a restoration of the intestinal microbiota to similar levels as healthy controls. The resultant microbiome composition has previously been shown to be associated with a reduction in the frequency of pulmonary exacerbations. ${ }^{43}$

Acute bacterial and viral infections cause changes in the underlying microbiota. Chronic bacterial colonization with known pathogens such as Pseudomonas aeruginosa and Haemophilus influenzae in CF has been associated with distinct lung microbiomes, dominated by Prevotella and Flavobacterium in the case of $P$. aeruginosa and Neisseria when $H$. influenzae is present. ${ }^{47}$ Similarly, the microbiome may play a role in determining susceptibility to viral infection. The pneumococcal vaccine, and depletion of Streptococcus pneumoniae in an in vitro model has been associated with a $31 \%$ reduction in viral respiratory tract infections. ${ }^{48}$ Molyneaux et al showed that rhinovirus infection in healthy subjects has little effect on the underlying lung microbiota when measured in induced sputum samples. They did find, however, that in patients with COPD, rhinovirus infection led to an outgrowth of certain pathogenic bacteria, mainly those in the Proteobacteria phylum including Haemophilus influenzae. ${ }^{22}$ In a murine model, infection with influenza virus was shown to lead to changes 
in the gut microbiome due to intestinal injury mediated by Th-17 cell-dependent inflammation. ${ }^{49}$

Murine studies have shown that bacterial activation of Nod-like receptors in the gut led to increased reactive oxygen species in alveolar macrophages ${ }^{50}$ which are associated with effective bacterial clearance mechanisms in the lung. Peptidoglycan (a bacterial cell wall component) can translocate from the gut into the blood stream and the bone marrow to cause systemic and organ specific effects after systematic presentation to the host immune system via Nod1 receptors. This can lead to increased killing of Streptococcus pneumoniae and Staphylococcus aureus. ${ }^{51}$ Studies have shown that lipopolysaccharides (LPS), another bacterial product, can reduce viral infection rates suggesting that development of the gut microbiota may play an important role in susceptibility to respiratory viral infections. In vitro, pre-stimulation of human macrophages with LPS led to an $80 \%$ reduction in RSV and influenza infections ${ }^{52}$, demonstrating the complex interplay between bacterial and viral species.

Inter-organ microbiome interactions are not only limited to the gut. Early establishment of a commensal nasopharyngeal microbiota appears to be protective of RSV-induced airway hyper-responsiveness. Mice were infected with RSV and subsequently treated with antibiotics which depleted Streptococcus viridans in the nasal passage associated with a strong immune response to the RSV infection with increased numbers of inflammatory lymphocytes, reduced $\mathrm{T}_{\text {regs }}$ and transforming growth factor-beta, and increased airway hyper-responsiveness. ${ }^{53}$ The interactions between the resident microbiomes within different organ systems are yet to be fully characterized, however for chronic respiratory disease sufferers, this area presents many possible future treatment avenues.

\section{Bacterial Studies}


Pathological changes that occur in chronic respiratory conditions place selection pressures on the lung microbiome. Resultant shifts in growth patterns of specific bacterial populations are invoked by increased mucus production, changes in oxygen availability ${ }^{54}$ and temperature ${ }^{55}$ in certain areas and an exacerbated inflammatory response leading to increased production of cytokines. ${ }^{56,57}$ Use of oxygen therapy, antibiotics and steroids in the treatment of these conditions may also impact the microbiome (Figure 1). As COPD, interstitial pulmonary fibrosis (IPF) and CF are the respiratory conditions for which lung transplantation is most commonly indicated, the microbiota associated variations observed in these diseases will be the focus of this chapter. All of these conditions demonstrate characteristic periods of 'exacerbations' or deterioration from the stable state. Emerging evidence indicates that these exacerbations may be associated with dysbiosis of the lung microbiota.

\section{COPD}

Multiple studies have investigated the lung microbiota of patients with COPD during stable periods when no evidence of lung infection could be observed. Several sample types including BAL, explanted lung tissue and expectorated sputum samples have been analyzed. These studies have revealed that the distal airways and alveoli in COPD patients contain a distinct microbiome which may prove relevant to the progression of the disease and the intermittent development of infectious exacerbations. ${ }^{11}$ Differences in the bacterial microbiome between individuals with COPD, non-COPD smokers and non-smoking controls have been observed. However, Erb-Downward et al did not demonstrate a distinct 'COPD' microbiome using sequencing techniques. Instead there was an extensive overlap in the bacterial communities between these three groups but decreased diversity of bacterial species in those with severe COPD. ${ }^{29,58}$ When the microbiome was characterised in lung tissue samples using PCR and terminal restriction fragment length polymorphism analysis, the microbiota seen in individuals with severe COPD differed from individuals with CF and mild COPD controls. ${ }^{29}$ An increase in microbiome diversity in COPD compared with healthy controls has also been demonstrated 
using sequencing methods, however diversity did not seem to correlate with disease severity. Species clustering did occur, however, with use of inhaled therapy including both bronchodilators and corticosteriods. ${ }^{59}$ Spatial heterogeneity of bacterial species within the lung was markedly increased in patients with severe COPD. ${ }^{58}$ These shifts in the microbiota in COPD may be explained in part by the decreased surface area of the lung due to alveolar wall breakdown as the disease progresses, which leaves less available terrain for the distribution of the microbiota. This may also be responsible for the increased bacterial burden observed in severe destructive lung diseases.

It has been postulated that changes in the microbiome may underlie exacerbations of COPD. A number of studies have compared the composition of the microbiome during stable periods with the composition during an exacerbation to determine if certain bacterial species are associated with worsening symptoms. Sethi et al compared the presence and load of the most common bacterial strains observed during an acute exacerbation and in the stable state of COPD. They found that change in bacterial load is unlikely to be the main mechanism involved in the occurrence of an exacerbation and suggested that host-pathogen interactions were more important. ${ }^{60} \mathrm{~A}$ study by Millares et al showed that colonization with Pseudomonas aeruginosa in severe COPD did not have significant effects on the biodiversity of the rest of the microbiome. Furthermore, during exacerbations, previous Pseudomonas colonization had little effect on the changes in microbes observed, compared to those who had never been colonized, including an increased abundance of Haemophilus, Streptococcus and Moraxella species. ${ }^{61}$ Furthermore, it has been observed that during an exacerbation, movement toward the Proteobacteria phylum, including detection of a range of non-typical COPD pathogens occurs. Treatment also appeared to be an important factor in microbiome shifts. Antibiotic treatment initially decreases Proteobacteria, but also induces overall microbiome suppression. Treatment with corticosteroids alone enriched further for members of the Proteobacteria phylum. ${ }^{62}$ 


\section{Idiopathic Pulmonary Fibrosis}

There are few studies of the microbiome in the interstitial lung diseases. However, recent trials suggest that the microbiota may also have an important role in mediating the development of IPF. A randomized controlled trial showed a decrease in respiratory infections and an all-cause mortality benefit for patients receiving cotrimoxazole ${ }^{63}$, while the PANTHER trial demonstrated that immunosuppression led to increased mortality in patients with IPF ${ }^{64} \mathrm{~A}$ limited number of studies have shown a role for the microbiota in predicting more severe disease and progression. ${ }^{65-68}$ It has also been shown that pneumolysin (pneumococcus-associated toxin) mediates fibrotic progression in animal models of alveolar epithelium injury. ${ }^{69}$ Therefore the presence of certain bacteria within the lung microbiota may predispose certain individuals to the development of IPF.

\section{Cystic Fibrosis}

It is generally accepted that pathogenic bacteria such as S.aureus and P.aeruginosa are commonly present within the lower airways of patients with CF and almost universally present by adolescence. ${ }^{70} \mathrm{CF}$ was one of the notable exceptions to the previous understanding that the lungs were sterile. Hence, CF research has led the field in microbiome-related studies in chronic lung diseases.

Microbial composition and diversity vary with disease progression. As patients develop more severe disease, bacterial community diversity has been shown to decrease significantly. However, in individuals with a mild lung disease phenotype, the bacterial community diversity remains stable over time. In both cases, microbial density remained steady. The most significant driver of a microbiome shift was antibiotic usage, however over time with increased usage, bacterial communities developed resistance to change..$^{23,25}$

Studies have suggested that treatment outcomes do not correlate with the susceptibility of strains of Pseudomonas aeruginosa to certain antibiotics. Two large trials have shown no 
association between the clinical response to antibiotics during pulmonary exacerbations and in vitro bacterial susceptibility to the chosen antibiotics. ${ }^{71,72}$ This suggests that a single organism may not be responsible for causing an exacerbation. Instead, complex microbiome interactions may be of greater importance.

Multiple studies in CF have shown that there is no change in bacterial burden or community diversity between samples taken during stable periods and at the time of exacerbations. It is instead suggested that an exacerbation occurs when there is an acute dysbiosis of the respiratory microbiome (acute shift in bacterial species) coupled with a disordered host

immune response. ${ }^{25,73-75}$ Price et al showed that the lung microbiota in CF had a complex microbial composition, which appeared to be unique to each patient analysed. This microbiome demonstrated resilience to exacerbation and antibiotic treatment with persistence of microbial species observed at multiple time points. ${ }^{76}$

Microbial interactions play an important role in CF exacerbation frequency and progression of the disease. Emerging research is examining the possibility of modifying the microbiome to improve disease-related outcomes for patients with CF. ${ }^{43,77}$

\section{Lung Transplantation}

Many host-specific and environmental factors have been shown to influence the human microbiota, however in this review, the focus will be on the impact of lung transplantation on respiratory microbiome dynamics and in contrast, how these variations in the lung microbiome affect transplant outcomes. Lung transplantation provides us with a unique opportunity to separate out host-specific, immune-related and extrinsic factors in shaping the microbiome after donor lungs have been transplanted into a new recipient. In effect, this is transplantation of the human respiratory microbiome. 
Lung transplantation is often the only viable option remaining for individuals with end-stage lung disease, providing patients with hope for an improved longevity and quality of life. However, compared with other solid organ transplantation, lung transplantation has the lowest long-term survival rates. Worldwide, the median survival time post bilateral lung transplant is 7.4 years as reported in the International Society for Heart and Lung Transplantation (ISHLT) 2017 Registry report compared with a median of 12.4 years for both living and deceased donor kidney transplantation. ${ }^{78}$

Lung transplantation is associated with multiple opportunities for lower respiratory tract sampling with both surveillance and clinically indicated bronchoscopies. Some of the first lung microbiome studies incorporating longitudinal analysis were performed in the transplant cohort, due to the ability to conduct serial invasive sampling for surveillance of the allograft. Borewicz et $\mathrm{al}^{79}$ showed in a small cohort that the lung microbiome in transplant patients is not very stable in the early post-transplant period with less than $10 \%$ of species retained over the three time points. Another cohort of lung transplant recipients underwent longitudinal sampling of their lower respiratory tract for up to 12 months, showing that bacterial diversity increased during the first 9 months post-transplant. This likely reflects development of a new stable state in the donor lungs. After 9-12 months post lung transplant, bacterial diversity began to decrease. ${ }^{80}$ Finally, Willner and colleagues ${ }^{81}$ surveyed 16 patients who had been transplanted for CF and found dynamic changes in the lung microbiome over time which was heavily influenced by antibiotic use. These preliminary data indicate that there are important fluctuations in both microbiota burden and diversity, especially in the early transplant period. Some associations between bacterial diversity and negative outcomes ${ }^{81,82}$ have been suggested, however limited data at this point restrict conclusions.

It has been previously thought that unanticipated donor transmission of infectious organisms is a rare, and dangerous event. ${ }^{83}$ Much work has focused on reducing the occurrence of transmission events, specifically of blood-borne viruses such as Human Immunodeficiency 
Virus (HIV) and Hepatitis B (HBV) and C (HCV) viruses. However, emerging evidence shows that the microbiome is transplanted from the donor into the recipient at the time of lung transplantation and that the composition of the donor microbiome may have important implications for transplant outcomes. The post-transplant lung microbiome may also be modified through a range of factors. These include nosocomial acquisition of microbes from the ICU while intubated and from contact with other infected individuals while in hospital, transmission from the native lung in recipients who receive a single lung transplant, aspiration of gastric organisms due to the increased burden of gastro-esophageal reflux disease (GERD) in transplant recipients ${ }^{84}$ and the impact of the upper respiratory tract microbiota (Figure 2). Little research currently addresses the origin of the microbes that induce the longitudinal changes in the microbiome that have been previously described. ${ }^{79-81}$ Further research studies and a greater understanding are needed to characterize implications for clinical management.

The vagus and other sympathetic nerves are transected at the hilum during lung transplantation causing loss of neural supply and complete denervation of the lungs in the early transplant period. This leads to decreased cough reflex, impaired ciliary beat frequency leading to impaired mucociliary clearance and implications for gastro-esophageal motility. ${ }^{85}$ Reduction in mechanical clearance mechanisms such as cough and the mucociliary escalator have important implications in the ability of the recipient to clear inhaled pathogens and allergens and therefore reduces the rate of elimination of species which is important in maintaining the 'steady state' airway microbiota. ${ }^{86}$ Furthermore, recipients receive high level induction and maintenance immunosuppression ${ }^{87}$ which reduces the ability of the immune system to respond and clear microbial species, further impacting the elimination aspect in determining the microbial balance.

The immigration of bacterial species into the lower respiratory tract is accelerated with gastroesophageal dysfunction and reflux, which lead to increased aspiration events. The incidence of GERD is significantly increased after lung transplantation, ${ }^{84}$ which has been 
shown to impact the composition of the lower respiratory tract microbiota. ${ }^{32}$ Furthermore, the use of acid-suppression medications such as proton-pump inhibitors have been associated with an increased risk of overgrowth of acid-sensitive gastric flora such as Streptococcus and Staphylococcus, which were also found at higher concentrations in the lungs following reflux events, indicating the likely exchange of microflora between these two sites. ${ }^{88}$ These bacteria are often associated with symptomatic upper respiratory tract infections and pneumonia. ${ }^{89}$ Laryngeal dysfunction and oral hygiene may also have an impact on lower respiratory tract microbiota due to increased pathogenic species in the oral cavity spreading to the airways, causing variations in the lung microbiome and through the increased risk of aspiration and inhalation events when there are alterations to the larynx. ${ }^{90,91}$ Furthermore, altered growth conditions within the lungs may have significant effects on microbiome dynamics with outgrowth of certain species and increased spatial heterogeneity within the lung field. These altered conditions may be caused by a number of factors including modifications to the airway terrain due to changes in ciliary function and mucus production, changes in regional growth conditions due to pneumocystis and general bacterial prophylaxis, and finally, effects of reperfusion after the transplant has occurred (Figure 2). ${ }^{37}$

Mortality within the first-year post-transplant is associated with primary graft failure and acute rejection events. Within the first year of transplantation, up to $55 \%$ of recipients are treated for acute allograft rejection. ${ }^{92}$ However, there has been limited research in acute rejection compared with chronic rejection and bronchiolitis obliterans syndrome (BOS), but there is some evidence to suggest that both bacterial and viral infections, and possibly perturbations to the underlying microbiota, may have an important role in mediating early allograft dysfunction. Glanville et al showed that persistent Chlamydia pneumoniae infection is associated with early mortality and rejection events. ${ }^{93}$ Parainfluenza virus (PIV) infection also has a demonstrated association with the development of acute allograft dysfunction, with one study showing that $82 \%$ of patients with parainfluenza viral infection in their cohort also had evidence of acute allograft dysfunction. Furthermore, $32 \%$ of these PIV positive patients 
developed bronchiolitis obliterans within 18 months follow up. ${ }^{94}$ Conversely, Ahya et al showed that detection of EBV DNA was not correlated with the development of acute allograft rejection. Instead, the authors suggested that increased viral load may be a surrogate marker of effective immunosuppression thus providing protective allograft effects. ${ }^{95}$

The principal factor limiting long-term survival in lung transplant recipients is chronic lung allograft dysfunction (CLAD) mostly due to BOS. According to the ISHLT Registry Report, 50\% of lung transplant recipients develop BOS within 5 years of transplant, while $76 \%$ of recipients develop BOS within 10 years. ${ }^{96}$ The primary pathological feature is bronchiolitis obliterans, a form of intraluminal airway fibrosis located mainly within the terminal bronchioles. ${ }^{97}$ It is now recognized that the causes of BOS are heterogeneous, and have differing clinical courses. Both viral and bacterial infections have been suggested to be risk factors for the development of BOS. ${ }^{98,99}$ New evidence emerging with the use of non-culture based techniques indicates that the underlying lung microbiome may play a greater role. ${ }^{8}$ Early studies investigating the respiratory microbiome post lung transplantation indicate that lung transplant recipients have variations in their lung microbiome compared with healthy controls ${ }^{100}$, and furthermore, that the microbiome changes over the post-transplant period ${ }^{79}$ as the lung microbiota becomes established within its new host and as an equilibrium is reached between the host immune response and immunosuppression levels. In these studies that have shown differences in transplant microbiome compared with healthy controls, one study has indicated increased bacterial burden in the BAL of lung transplant recipients ${ }^{79}$, while in others, decreased bacterial burden was observed in transplanted lungs. ${ }^{81,82,100}$ Some suggestions of decreased community diversity were seen. However, this appears to more commonly be the case in those with active respiratory symptoms and is not necessarily observed in asymptomatic controls. ${ }^{82}$ Therefore, further information about the differences between symptomatic and asymptomatic transplant patients are required including elucidating how these may correlate with both microbiome dysbiosis and negative outcomes in this cohort. 
A number of studies have investigated the relationship between the presence of specific members of the microbiome and the risk of BOS. This relationship was first demonstrated using traditional culture-based techniques and serum antibody detection. More recently the advent of metagenomics techniques has allowed the dynamics of the greater lung microbiome to be examined in more detail. Some bacterial species, such as Pseudomonas appear to play a greater role in mediating the development of BOS. Multiple culture based studies have consistently shown that airway colonization with $P$. aeruginosa is predictive of subsequent

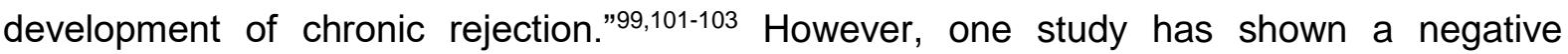
correlation between presence of Pseudomonas spp. and development of BOS. ${ }^{81}$ In those studies where a positive relationship between Pseudomonas colonization, and development of BOS was seen, de novo colonization post-transplant rather than persistence of pretransplant Pseudomonas colonization had an increased likelihood of BOS development. ${ }^{81,103}$

Patients with suppurative lung disease, including CF, who had chronic colonization prior to transplant are at a greater risk of re-infecting the allograft with persistent bacterial species from the upper airway and the sinuses (Figure 2). Vital et al attempted to mitigate this risk by performing endoscopic frontal-spheno-ethmoidectomy procedures immediately posttransplant and using multiple Pseudomonas-active antibiotics. Despite this treatment, $65 \%$ of patients had persistent colonization post-transplantation. This study also demonstrated greater BOS-free survival in those patients who did not demonstrate detection of Pseudomonas. ${ }^{104}$ Dickson et al identified two distinct Pseudomonas species which were both prominent in the lung microbiome samples of lung transplant recipients, but were associated with distinct clinical phenotypes. P. aeruginosa, when detected, is usually seen in high abundance and is accompanied by acute clinical infection, whereas $P$. fluorescens is usually detected at a moderate abundance and is rarely correlated with development of acute symptomatic infection. ${ }^{82}$ If the bacteria are only identified at the family level, the distinction between these species may be missed. $P$. fluorescens has been shown to be rarely detected using culture-based methodologies, thus the authors suggest this may account for the lack of 
correlation between detection of Pseudomonas and development of BOS seen in the study by Willner et al, who utilised culture-independent techniques. ${ }^{81}$ Therefore, characterization of the microbiome and subsequent monitoring may provide further information on risk of BOS. It also highlights the future possibility of modifying the microbiome in attempts to reduce the development of BOS.

The increased access to culture independent techniques has played an important part in defining the role of the respiratory microbiome in lung transplantation. Variations in the lung microbiome post-transplant appear to modify the risk of both acute and chronic rejection events. Lung transplantation provides us with a unique scenario in which to investigate the lung microbiome and the changes that may occur in response to a new host, surgery-related damage and donor-transmission events. The substantial follow-up opportunities presented have allowed the lower respiratory tract microbiome to be monitored longitudinally and for the dynamics and shift of microbial species to begin to be elucidated.

\section{Fungal Studies}

Compared with the bacterial component of the lung microbiome, the fungal component termed the 'mycobiome' represents a largely understudied area with fewer than 10 published papers describing the fungal composition of the microbiome using next generation sequencing techniques (NGS). Despite the limited data present in this area, there appears to be many parallels between the fungal and bacterial components of the microbiome including the understanding that fungal species are present during health, and that there is significant intrasubject variability in the diversity of species detected. ${ }^{105}$ Limited studies have compared fungal and bacterial species in disease states with healthy controls. The most common fungal genera identified in healthy lung samples were Cladosporium, Eurotium, Penicillium, Aspergillus,

Candida, and Pneumocystis. ${ }^{100,106}$ Similarly to the bacterial component, a large range of fungal species have been found in lung samples since the use of NGS, which are not detectable by 
culture. Up to $82 \%$ of the fungal species detected in sputum samples by sequencing were unable to be cultured. Furthermore, in this group of 55 CF patients, only $27 \%$ were found to be positive for fungus when culture-based methods were used, compared with $90 \%$ of the cohort being positive by sequencing. ${ }^{107}$

The lung mycobiome has been investigated in CF and COPD, with decreases in fungal diversity being observed. Furthermore, decreased fungal diversity has been associated with lower lung function. It has been suggested that the decreased diversity may be due to outgrowth of a single species, or the loss of rare species as part of overall decreased fungal abundance. In CF, it has been shown that the highest species richness is observed in those with the lowest disease severity scores. ${ }^{106}$ Similarly to the observations from the bacterial component of the microbiome, fungal communities also appear to remain stable through antimicrobial therapy and exacerbations. ${ }^{108,109}$ There are very limited data regarding the mycobiome in COPD, however two studies have shown Aspergillus ${ }^{110}$ and Pneumocystis ${ }^{111}$ to be highly represented species within BAL samples from patients with COPD. How these interact with other fungal species, and other bacterial members of the microbiome is yet to be elucidated.

Many aspects impact the microbiome in transplant, including donor transmission, structural changes within the lungs and immunosuppression. However, there is still limited information regarding how these factors impact the mycobiome. The fungal aspect of the microbiome has been characterised in a small set of lung transplant patients showing that in general, there is decreased fungal abundance and diversity compared with healthy controls. Charlson et al characterised the lung mycobiome in 21 patients following lung transplantation, and found that in many cases, it was largely dominated by Candida species which were also found in the oropharyngeal wash of these subjects. In two individuals, high levels of Aspergillus were detected, however these were largely absent in the oropharyngeal wash samples. Furthermore, Cryptococcus was observed in the BAL samples of 6 patients at relatively low 
abundance. ${ }^{100}$ Indication for transplant also appears to have an effect. Individuals transplanted for CF showed the lowest species richness. ${ }^{100}$

Certain members of the mycobiome may be associated with negative outcomes posttransplant. Colonization with Aspergillus has been shown to be strongly associated with development of BOS and increased BOS-related mortality using Cox-regression analyses. ${ }^{112}$ Willner et al demonstrated that Aspergillus species were decreased in transplant patients who had Pseudomonas-dominated microbiomes, which was shown to be negatively correlated with the development of BOS. ${ }^{81}$ Therefore, further characterization of the mycobiome is necessary to determine the contribution of members to negative outcomes post lung transplantation. This may contribute to long-term treatment strategies in microbiome manipulation as a way of mitigating the development and progression of BOS.

\section{Virome Studies}

There are limited data in the area of the virome due to the inherent limitations in isolating and defining this part of the microbiome. In the lungs, the bacterial biomass is reduced compared with other compartments such as the intestine and the oral cavity. The amount of viral genomic material is even lower. Isolating viral nucleic acids without the use of a conserved sequence such as $16 \mathrm{~S}$ in bacteria or ITS region for fungi, and effectively depleting both human and bacterial genomic material presents many challenges and may account for the lag in our knowledge of the viral component of the microbiome.

The first studies in the area of the lung virome were in patients with CF, paralleling the early studies of the bacterial component of the microbiome. Bacteriophage communities in a small cohort of individuals with CF were compared with healthy controls, to demonstrate that there appears to be a core set of bacteriophages found in the healthy lung and an additional core set of phages found in individuals with CF which correspond to concurrent pathogenic bacterial 
species. In CF, the lung bacteriophage community appears to be driven by airway pathology and the persistence of certain bacteria. ${ }^{113}$ The same group conducted a small case series evaluating spatial heterogeneity within CF lungs. A large range of DNA viruses and bacteriophages were observed within the lungs of individuals with end-stage lung disease, with clear heterogeneity in the presence of viruses within different lobes of the lungs. ${ }^{38}$ Since then, a handful of studies have largely characterized the virome in individuals with acute viral infections. In this case, there is an overwhelming abundance of the causative organism which is often concurrently detected using PCR methods. However, a range of other viral species including torque teno viruses, herpes viruses and community acquired respiratory viruses such as picornavirus and adenovirus have been characterised in these samples. ${ }^{114,115}$ Furthermore, a study characterizing the respiratory virome in febrile and afebrile children has provided evidence to show that a resident lung virome is likely established in healthy children within the first two years of life which may also impact the development of the immune system. ${ }^{116}$

The human respiratory virome has been investigated in lung transplantation. Young et al have characterized the DNA virus profile in the oropharyngeal washes and BAL samples posttransplant. ${ }^{117}$ A range of DNA viruses were detected using sequencing, including herpes viruses, human papillomavirus and bacteriophages. Anelloviruses, including torque teno virus, dominated these samples accounting for over $68 \%$ of reads. These anelloviruses were further quantified using real time qPCR. Transplant patients were shown to have significantly greater viral loads in both the oropharyngeal wash and BAL samples compared to healthy controls. ${ }^{117}$ A further study explored the role of torque teno virus in lung transplant recipients in the perioperative period. Torque teno virus was shown to increase in the immediate post-operative period, however the magnitude of viral load increase was shown to be associated with development of primary graft dysfunction. ${ }^{118}$ Therefore, monitoring changes in the virome may, in the future, assist in determining risk profiles for graft injury and adequate levels of immunosuppression to guide therapeutic actions post-transplantation (Figure 3). 
There is some evidence to suggest that viral infections are associated with the development of BOS. Garantziotis et al showed a relationship between infection with influenza and subsequent decline in lung function associated with early graft dysfunction and / or BOS. ${ }^{119}$ A further study has indicated that the one-year incidence of BOS is significantly increased $(25 \%)$ in patients who have had a respiratory virus detected by PCR compared with virusnegative patients $(9 \%)$. However, detection of a respiratory virus in patients who already had diagnosed BOS had no impact on progression. ${ }^{120}$ This was further evidenced by a retrospective 5-year cohort study on lung transplant patients, where detection of a lower respiratory tract viral infection was significantly correlated with development of BOS and BOSrelated mortality. ${ }^{121} \mathrm{~A}$ further study that compared patients with symptomatic viral infection with asymptomatic controls, demonstrated PCR detected virus in the symptomatic group which was associated with both acute rejection events and progression to BOS. ${ }^{122}$

The herpes group viruses have been studied extensively as a quasi-resident species within the lungs of transplant recipients. Cytomegalovirus (CMV) and Epstein Barr Virus (EBV) as well as Human Herpesvirus Type 6 (HHV-6), in particular, have received attention but data regarding their relationship to allograft dysfunction are somewhat confounded by the efficacy of prophylactic use of specific antiviral therapies. ${ }^{123}$ Some relationships are clear. An EBV naïve recipient of an EBV positive lung allograft has the highest risk of developing posttransplant lymphoproliferative disease (PTLD) (50\% in some series) which carries a high mortality rate. This has been somewhat ameliorated of recent times by the selective use of anti-CD20 monoclonal antibody therapy to target EBV driven B-cell clonal expansion as an adjunct to reduction in immune suppression which, of itself, was commonly associated with the development of acute cellular rejection and graft dysfunction. This relationship between the risk of PTLD and EBV viral load has led some units to pursue a policy of peripheral load monitoring in blood using a quantitative EBV PCR with the intention of reducing immune suppression pre-emptively if EBV load increases, to circumvent the risk of PTLD. ${ }^{124}$ 
Conversely an absent EBV load raises the question of acute rejection risk. The oncogenic potential of EBV, Kaposi's sarcoma herpesvirus (KSHV), and human T-lymphotropic virus type 1 (HTLV-1) has been confirmed by high-throughput RNA sequencing data from 50 common lymphoma cell culture models from the Cancer Cell Line Encyclopedia project. ${ }^{125}$ The role of HHV-6 in the pathogenesis of organizing pneumonia, a not uncommon finding on transbronchial biopsy deserves further study. ${ }^{123}$ Repeated EBV DNA detection in blood, possibly reflecting chronic EBV replication, has been associated with the development of BOS. ${ }^{126}$

CMV can cause fulminant pneumonia and death, which since the development of effective treatment modalities ${ }^{127}$ is now rare, except for drug resistant strains, an ever-present risk of the use of widespread prophylaxis. ${ }^{128} \mathrm{~A}$ largely intracellular virus, CMV can be detected in bronchoalveolar lavage fluid and analysis of paired samples suggests detection of CMV DNA in BAL fluid reflects virus replication in the lung rather than oropharyngeal contamination. ${ }^{129}$ Whether BAL load monitoring assists in the determination of a therapeutic decision is debatable versus a qualitative assessment. However, peripheral load monitoring in blood using a quantitative PCR is the gold standard for initiation of therapy versus prophylaxis. CMV naïve recipients of a positive donor graft hold the highest risk of CMV pneumonia, therefore there exists some evidence for ongoing prophylaxis. ${ }^{130}$

To date, there are very limited studies characterizing the human virome, and none in the areas of COPD or IPF. Further research may help to elucidate the changes in the resident virome between different disease states, and how these impact progression. Early studies in lung transplantation show distinct differences in the respiratory virome composition and diversity between lung transplant recipients and healthy controls. However, the detection of causative viral organisms in symptomatic individuals has been associated with negative transplant outcomes. The presence of certain viral species, and their viral load may help to guide treatment strategies and modifications to the respiratory virome may improve outcomes for 
patients in the future (Figure 3). In the majority of lung virome studies published, sequencing has focused on the DNA virus community due to the inherent limitations of working with RNA viruses which are much more prone to degradation. Little is known about the role of RNA viruses in both healthy individuals and in disease states, including after transplantation. Further studies, particularly those with a prospective longitudinal design, are needed to describe the large collection of viruses detected by NGS which remain unnamed and uncharacterized and to determine their role within the greater microbiome in both health and disease.

\section{Summary and Conclusions}

In a very real sense, our understanding of the component parts of the microbiome and, in particular, the human respiratory microbiome is just beginning. New tools, including next generation sequencing are now available to assist in the exploration of this exciting field of knowledge, however much work remains. Essentially, we need to define the constituents of the human respiratory microbiome in health and in disease states. Furthermore, we need to develop an understanding of the range of normal throughout different phases of life and between different communities. In the human respiratory virome, in particular, it is vital to appreciate the concept of resident species such as anelloviruses and the impact of transient species such as community acquired respiratory viruses to underpin future research given the ubiquitous nature of the latter. Mapping the spatial architecture within the lung could also prove critical in localizing pathology to lung regions and providing potential targeted therapeutic options. Similarly, the ability to detect pathological alterations in the respiratory microbiome will depend on a deep understanding of the dynamics and inter-relationships between bacteria, viruses, fungi and archaea. The study of these relationships will perhaps become a field unto itself. To date, there are little data in this area as a silo mentality has prevailed which has allowed a focus on one component at a time, perhaps reflecting individual interests and experience, as well as the limits of technology available, not to mention financial constraints. 
The challenge, therefore, for funding bodies especially, is to see and appreciate the whole, and not just the component parts. Only then, will the workings of the integrated machine become clear and our contemporary knowledge, which has been beset by false premises of microbial sterility of the lung, be cast aside to permit a revised vision of the complex microbial basis of this precious organ that allows us to breathe. Transplanting the human respiratory microbiome, as perforce is integral to lung transplantation, is a bold endeavor replete with its own special challenges where early exploratory evidence based on serial monitoring is showing promise of recognizing causes and outcomes of dysbiosis related to immune suppression. Whether real time analysis will permit therapeutic adjustments to facilitate better outcomes remains to be tested in the crucible of clinical practice. 


\section{References}

1. Gill SR, Pop M, Deboy RT, et al. Metagenomic analysis of the human distal gut microbiome. Science 2006;312:1355-9.

2. Backhed F, Ley RE, Sonnenburg JL, Peterson DA, Gordon JI. Host-bacterial mutualism in the human intestine. Science 2005;307:1915-20.

3. Turnbaugh PJ, Ley RE, Hamady M, Fraser-Liggett CM, Knight R, Gordon JI. The human microbiome project. Nature 2007;449:804-10.

4. Group NHW, Peterson J, Garges S, et al. The NIH Human Microbiome Project. Genome Res 2009;19:2317-23.

5. DeGruttola AK, Low D, Mizoguchi A, Mizoguchi E. Current Understanding of Dysbiosis in Disease in Human and Animal Models. Inflamm Bowel Dis 2016;22:1137-50.

6. Clarridge JE, 3rd. Impact of $16 \mathrm{~S}$ rRNA gene sequence analysis for identification of bacteria on clinical microbiology and infectious diseases. Clin Microbiol Rev 2004;17:840-62, table of contents.

7. Schuster SC. Next-generation sequencing transforms today's biology. Nat Methods 2008;5:16-8.

8. Becker J, Poroyko V, Bhorade S. The lung microbiome after lung transplantation. Expert Rev Respir Med 2014;8:221-31.

9. Gleeson K, Eggli DF, Maxwell SL. Quantitative aspiration during sleep in normal subjects. Chest 1997;111:1266-72.

10. Huxley EJ, Viroslav J, Gray WR, Pierce AK. Pharyngeal aspiration in normal adults and patients with depressed consciousness. Am J Med 1978;64:564-8.

11. Dickson RP, Erb-Downward JR, Huffnagle GB. The role of the bacterial microbiome in lung disease. Expert Rev Respir Med 2013;7:245-57.

12. The Lung HIV Microbiome Project (LHMP). National Heart, Lung and Blood Institute, 2015. (Accessed June 30, 2017, at https://biolincc.nhlbi.nih.gov/studies//hmp/.)

13. Mackie RI, Sghir A, Gaskins HR. Developmental microbial ecology of the neonatal gastrointestinal tract. Am J Clin Nutr 1999;69:1035S-45S.

14. DiGiulio DB. Diversity of microbes in amniotic fluid. Semin Fetal Neonatal Med 2012;17:2-11.

15. Aagaard K, Ma J, Antony KM, Ganu R, Petrosino J, Versalovic J. The placenta harbors a unique microbiome. Sci Transl Med 2014;6:237ra65.

16. Stout MJ, Conlon B, Landeau M, et al. Identification of intracellular bacteria in the basal plate of the human placenta in term and preterm gestations. Am J Obstet Gynecol 2013;208:226 e1-7.

17. Gollwitzer ES, Saglani S, Trompette A, et al. Lung microbiota promotes tolerance to allergens in neonates via PD-L1. Nat Med 2014;20:642-7.

18. Teo SM, Mok D, Pham K, et al. The infant nasopharyngeal microbiome impacts severity of lower respiratory infection and risk of asthma development. Cell Host Microbe 2015;17:704-15.

19. Biesbroek G, Tsivtsivadze E, Sanders EA, et al. Early respiratory microbiota composition determines bacterial succession patterns and respiratory health in children. Am J Respir Crit Care Med 2014;190:1283-92.

20. Rogers GB, van der Gast CJ, Cuthbertson L, et al. Clinical measures of disease in adult non-CF bronchiectasis correlate with airway microbiota composition. Thorax 2013;68:731-7.

21. Rogers GB, Zain NM, Bruce KD, et al. A novel microbiota stratification system predicts future exacerbations in bronchiectasis. Ann Am Thorac Soc 2014;11:496-503.

22. Molyneaux PL, Mallia P, Cox MJ, et al. Outgrowth of the bacterial airway microbiome after rhinovirus exacerbation of chronic obstructive pulmonary disease. Am J Respir Crit Care Med 2013;188:1224-31.

23. Cox MJ, Allgaier M, Taylor B, et al. Airway microbiota and pathogen abundance in agestratified cystic fibrosis patients. PLoS One 2010;5:e11044. 
24. Zhao J, Murray S, Lipuma JJ. Modeling the impact of antibiotic exposure on human microbiota. Sci Rep 2014;4:4345.

25. Zhao J, Schloss PD, Kalikin LM, et al. Decade-long bacterial community dynamics in cystic fibrosis airways. Proc Natl Acad Sci U S A 2012;109:5809-14.

26. Goddard AF, Staudinger BJ, Dowd SE, et al. Direct sampling of cystic fibrosis lungs indicates that DNA-based analyses of upper-airway specimens can misrepresent lung microbiota. Proc Natl Acad Sci U S A 2012;109:13769-74.

27. Bassis CM, Erb-Downward JR, Dickson RP, et al. Analysis of the upper respiratory tract microbiotas as the source of the lung and gastric microbiotas in healthy individuals. MBio 2015;6:e00037.

28. Segal LN, Alekseyenko AV, Clemente JC, et al. Enrichment of lung microbiome with supraglottic taxa is associated with increased pulmonary inflammation. Microbiome 2013;1:19. 29. Sze MA, Dimitriu PA, Hayashi $S$, et al. The lung tissue microbiome in chronic obstructive pulmonary disease. Am J Respir Crit Care Med 2012;185:1073-80.

30. West JB. Regional differences in the lung. Chest 1978;74:426-37.

31. O'Dwyer DN, Dickson RP, Moore BB. The Lung Microbiome, Immunity, and the Pathogenesis of Chronic Lung Disease. J Immunol 2016;196:4839-47.

32. Dickson RP, Erb-Downward JR, Martinez FJ, Huffnagle GB. The Microbiome and the Respiratory Tract. Annu Rev Physiol 2016;78:481-504.

33. Bidan CM, Veldsink AC, Meurs H, Gosens R. Airway and Extracellular Matrix Mechanics in COPD. Front Physiol 2015;6:346.

34. Postma DS, Timens W. Remodeling in asthma and chronic obstructive pulmonary disease. Proc Am Thorac Soc 2006;3:434-9.

35. Dickson RP, Erb-Downward JR, Prescott HC, et al. Analysis of culture-dependent versus culture-independent techniques for identification of bacteria in clinically obtained bronchoalveolar lavage fluid. J Clin Microbiol 2014;52:3605-13.

36. Hilty $\mathrm{M}$, Burke $\mathrm{C}$, Pedro $\mathrm{H}$, et al. Disordered microbial communities in asthmatic airways. PLoS One 2010;5:e8578.

37. Dickson RP, Erb-Downward JR, Freeman CM, et al. Spatial Variation in the Healthy Human Lung Microbiome and the Adapted Island Model of Lung Biogeography. Ann Am Thorac Soc 2015;12:821-30.

38. Willner D, Haynes MR, Furlan M, et al. Case studies of the spatial heterogeneity of DNA viruses in the cystic fibrosis lung. Am J Respir Cell Mol Biol 2012;46:127-31.

39. Madan JC, Koestler DC, Stanton BA, et al. Serial analysis of the gut and respiratory microbiome in cystic fibrosis in infancy: interaction between intestinal and respiratory tracts and impact of nutritional exposures. MBio 2012;3.

40. Trompette A, Gollwitzer ES, Yadava K, et al. Gut microbiota metabolism of dietary fiber influences allergic airway disease and hematopoiesis. Nat Med 2014;20:159-66.

41. Inagaki H, Suzuki T, Nomoto K, Yoshikai Y. Increased susceptibility to primary infection with Listeria monocytogenes in germfree mice may be due to lack of accumulation of L-selectin+ CD44+ T cells in sites of inflammation. Infect Immun 1996;64:3280-7.

42. Abrahamsson TR, Jakobsson HE, Andersson AF, Bjorksten B, Engstrand L, Jenmalm MC. Low diversity of the gut microbiota in infants with atopic eczema. J Allergy Clin Immunol 2012;129:43440, 40 e1-2.

43. Bruzzese E, Callegari ML, Raia V, et al. Disrupted intestinal microbiota and intestinal inflammation in children with cystic fibrosis and its restoration with Lactobacillus GG: a randomised clinical trial. PLoS One 2014;9:e87796.

44. Bisgaard H, Li N, Bonnelykke K, et al. Reduced diversity of the intestinal microbiota during infancy is associated with increased risk of allergic disease at school age. J Allergy Clin Immunol 2011;128:646-52 e1-5. 
45. Oyama $N$, Sudo $N$, Sogawa $H$, Kubo $C$. Antibiotic use during infancy promotes a shift in the

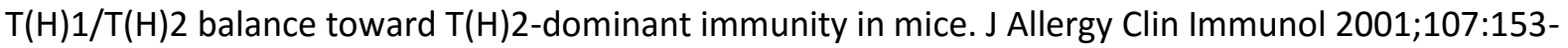
9.

46. Russell SL, Gold MJ, Willing BP, Thorson L, McNagny KM, Finlay BB. Perinatal antibiotic treatment affects murine microbiota, immune responses and allergic asthma. Gut Microbes 2013;4:158-64.

47. Rogers GB, van der Gast CJ, Serisier DJ. Predominant pathogen competition and core microbiota divergence in chronic airway infection. ISME J 2015;9:217-25.

48. Dagan R, Sikuler-Cohen M, Zamir O, Janco J, Givon-Lavi N, Fraser D. Effect of a conjugate pneumococcal vaccine on the occurrence of respiratory infections and antibiotic use in day-care center attendees. Pediatr Infect Dis J 2001;20:951-8.

49. Wang J, Li F, Wei H, Lian ZX, Sun R, Tian Z. Respiratory influenza virus infection induces intestinal immune injury via microbiota-mediated Th17 cell-dependent inflammation. J Exp Med 2014;211:2397-410.

50. Clarke TB. Early innate immunity to bacterial infection in the lung is regulated systemically by the commensal microbiota via nod-like receptor ligands. Infect Immun 2014;82:4596-606.

51. Clarke TB, Davis KM, Lysenko ES, Zhou AY, Yu Y, Weiser JN. Recognition of peptidoglycan from the microbiota by Nod1 enhances systemic innate immunity. Nat Med 2010;16:228-31.

52. Short KR, Vissers M, de Kleijn S, et al. Bacterial lipopolysaccharide inhibits influenza virus infection of human macrophages and the consequent induction of CD8+ T cell immunity. J Innate Immun 2014;6:129-39.

53. Ni K, Li S, Xia Q, et al. Pharyngeal microflora disruption by antibiotics promotes airway hyperresponsiveness after respiratory syncytial virus infection. PLoS One 2012;7:e41104.

54. Worlitzsch $D$, Tarran $R$, Ulrich $M$, et al. Effects of reduced mucus oxygen concentration in airway Pseudomonas infections of cystic fibrosis patients. J Clin Invest 2002;109:317-25.

55. Schmidt A, Belaaouaj A, Bissinger R, et al. Neutrophil elastase-mediated increase in airway temperature during inflammation. J Cyst Fibros 2014;13:623-31.

56. Kanangat $S$, Meduri GU, Tolley EA, et al. Effects of cytokines and endotoxin on the intracellular growth of bacteria. Infect Immun 1999;67:2834-40.

57. Meduri GU, Kanangat S, Stefan J, Tolley E, Schaberg D. Cytokines IL-1beta, IL-6, and TNFalpha enhance in vitro growth of bacteria. Am J Respir Crit Care Med 1999;160:961-7.

58. Erb-Downward JR, Thompson DL, Han MK, et al. Analysis of the lung microbiome in the "healthy" smoker and in COPD. PLoS One 2011;6:e16384.

59. Pragman AA, Kim HB, Reilly CS, Wendt C, Isaacson RE. The lung microbiome in moderate and severe chronic obstructive pulmonary disease. PLoS One 2012;7:e47305.

60. Sethi S, Sethi R, Eschberger K, et al. Airway bacterial concentrations and exacerbations of chronic obstructive pulmonary disease. Am J Respir Crit Care Med 2007;176:356-61.

61. Millares L, Ferrari R, Gallego M, et al. Bronchial microbiome of severe COPD patients colonised by Pseudomonas aeruginosa. Eur J Clin Microbiol Infect Dis 2014;33:1101-11.

62. Huang YJ, Sethi S, Murphy T, Nariya S, Boushey HA, Lynch SV. Airway microbiome dynamics in exacerbations of chronic obstructive pulmonary disease. J Clin Microbiol 2014;52:2813-23.

63. Shulgina L, Cahn AP, Chilvers ER, et al. Treating idiopathic pulmonary fibrosis with the addition of co-trimoxazole: a randomised controlled trial. Thorax 2013;68:155-62.

64. Idiopathic Pulmonary Fibrosis Clinical Research N, Raghu G, Anstrom KJ, King TE, Jr., Lasky JA, Martinez FJ. Prednisone, azathioprine, and N-acetylcysteine for pulmonary fibrosis. N Engl J Med 2012;366:1968-77.

65. Molyneaux PL, Cox MJ, Willis-Owen SA, et al. The role of bacteria in the pathogenesis and progression of idiopathic pulmonary fibrosis. Am J Respir Crit Care Med 2014;190:906-13.

66. Molyneaux PL, Maher TM. Respiratory microbiome in IPF: cause, effect, or biomarker? Lancet Respir Med 2014;2:511-3. 
67. Salisbury ML, Han MK, Dickson RP, Molyneaux PL. Microbiome in interstitial lung disease: from pathogenesis to treatment target. Curr Opin Pulm Med 2017.

68. Han MK, Zhou Y, Murray S, et al. Lung microbiome and disease progression in idiopathic pulmonary fibrosis: an analysis of the COMET study. Lancet Respir Med 2014;2:548-56.

69. Knippenberg S, Ueberberg B, Maus $R$, et al. Streptococcus pneumoniae triggers progression of pulmonary fibrosis through pneumolysin. Thorax 2015;70:636-46.

70. Ramsey BW. Management of pulmonary disease in patients with cystic fibrosis. N Engl J Med 1996;335:179-88.

71. Hurley MN, Ariff AH, Bertenshaw C, Bhatt J, Smyth AR. Results of antibiotic susceptibility testing do not influence clinical outcome in children with cystic fibrosis. J Cyst Fibros 2012;11:288-

92.

72. Smith AL, Fiel SB, Mayer-Hamblett N, Ramsey B, Burns JL. Susceptibility testing of Pseudomonas aeruginosa isolates and clinical response to parenteral antibiotic administration: lack of association in cystic fibrosis. Chest 2003;123:1495-502.

73. Carmody LA, Zhao J, Kalikin LM, et al. The daily dynamics of cystic fibrosis airway microbiota during clinical stability and at exacerbation. Microbiome 2015;3:12.

74. Carmody LA, Zhao J, Schloss PD, et al. Changes in cystic fibrosis airway microbiota at pulmonary exacerbation. Ann Am Thorac Soc 2013;10:179-87.

75. Stressmann FA, Rogers GB, Marsh P, et al. Does bacterial density in cystic fibrosis sputum increase prior to pulmonary exacerbation? J Cyst Fibros 2011;10:357-65.

76. Price $\mathrm{KE}$, Hampton TH, Gifford $\mathrm{AH}$, et al. Unique microbial communities persist in individual cystic fibrosis patients throughout a clinical exacerbation. Microbiome 2013;1:27.

77. Weiss B, Bujanover Y, Yahav Y, Vilozni D, Fireman E, Efrati O. Probiotic supplementation affects pulmonary exacerbations in patients with cystic fibrosis: a pilot study. Pediatr Pulmonol 2010;45:536-40.

78. Rana A, Gruessner A, Agopian VG, et al. Survival benefit of solid-organ transplant in the United States. JAMA Surg 2015;150:252-9.

79. Borewicz K, Pragman AA, Kim HB, Hertz M, Wendt C, Isaacson RE. Longitudinal analysis of the lung microbiome in lung transplantation. FEMS Microbiol Lett 2013;339:57-65.

80. Luna R, Sagar M, Crabtree $S$, et al. Characterization of the lung microbiome in pediatric lung transplant recipients. The Journal of Heart and Lung Transplantation 2013;32:S291.

81. Willner DL, Hugenholtz P, Yerkovich ST, et al. Reestablishment of recipient-associated microbiota in the lung allograft is linked to reduced risk of bronchiolitis obliterans syndrome. Am J Respir Crit Care Med 2013;187:640-7.

82. Dickson RP, Erb-Downward JR, Freeman CM, et al. Changes in the lung microbiome following lung transplantation include the emergence of two distinct Pseudomonas species with distinct clinical associations. PLoS One 2014;9:e97214.

83. Ison MG, Hager J, Blumberg E, et al. Donor-derived disease transmission events in the United States: data reviewed by the OPTN/UNOS Disease Transmission Advisory Committee. Am J Transplant 2009;9:1929-35.

84. Young LR, Hadjiliadis D, Davis RD, Palmer SM. Lung transplantation exacerbates gastroesophageal reflux disease. Chest 2003;124:1689-93.

85. Ferdinande P, Bruyninckx F, Van Raemdonck D, Daenen W, Verleden G, Leuven Lung Transplant $G$. Phrenic nerve dysfunction after heart-lung and lung transplantation. J Heart Lung Transplant 2004;23:105-9.

86. Herve P, Silbert D, Cerrina J, Simonneau G, Dartevelle P. Impairment of bronchial mucociliary clearance in long-term survivors of heart/lung and double-lung transplantation. The Paris-Sud Lung Transplant Group. Chest 1993;103:59-63.

87. Bhorade SM, Villanueva J, Jordan A, Garrity ER. Immunosuppressive regimens in lung transplant recipients. Drugs Today (Barc) 2004;40:1003-12. 
88. Rosen $\mathrm{R}$, Amirault J, Liu $\mathrm{H}$, et al. Changes in gastric and lung microflora with acid suppression: acid suppression and bacterial growth. JAMA Pediatr 2014;168:932-7.

89. Dasaraju PV, Liu C. Infections of the Respiratory System. In: Baron S, ed. Medical Microbiology. 4th ed. Galveston (TX)1996.

90. Avila $\mathrm{M}$, Ojcius DM, Yilmaz $\mathrm{O}$. The oral microbiota: living with a permanent guest. DNA Cell Biol 2009;28:405-11.

91. Kenn K, Hess MM. Vocal cord dysfunction: an important differential diagnosis of bronchial asthma. Dtsch Arztebl Int 2008;105:699-704.

92. Martinu T, Chen DF, Palmer SM. Acute rejection and humoral sensitization in lung transplant recipients. Proc Am Thorac Soc 2009;6:54-65.

93. Glanville AR, Gencay M, Tamm M, et al. Chlamydia pneumoniae infection after lung transplantation. J Heart Lung Transplant 2005;24:131-6.

94. Vilchez RA, McCurry K, Dauber J, et al. The epidemiology of parainfluenza virus infection in lung transplant recipients. Clin Infect Dis 2001;33:2004-8.

95. Ahya VN, Douglas LP, Andreadis C, et al. Association between elevated whole blood EpsteinBarr virus (EBV)-encoded RNA EBV polymerase chain reaction and reduced incidence of acute lung allograft rejection. J Heart Lung Transplant 2007;26:839-44.

96. Yusen RD, Christie JD, Edwards LB, et al. The Registry of the International Society for Heart and Lung Transplantation: Thirtieth Adult Lung and Heart-Lung Transplant Report--2013; focus theme: age. J Heart Lung Transplant 2013;32:965-78.

97. Thompson BR, Hodgson YM, Kotsimbos T, et al. Bronchiolitis obliterans syndrome leads to a functional deterioration of the acinus post lung transplant. Thorax 2014;69:487-8.

98. Billings JL, Hertz MI, Savik K, Wendt $\mathrm{CH}$. Respiratory viruses and chronic rejection in lung transplant recipients. J Heart Lung Transplant 2002;21:559-66.

99. Vos R, Vanaudenaerde BM, Geudens N, Dupont LJ, Van Raemdonck DE, Verleden GM. Pseudomonal airway colonisation: risk factor for bronchiolitis obliterans syndrome after lung transplantation? Eur Respir J 2008;31:1037-45.

100. Charlson ES, Diamond JM, Bittinger K, et al. Lung-enriched organisms and aberrant bacterial and fungal respiratory microbiota after lung transplant. Am J Respir Crit Care Med 2012;186:536-45. 101. Gottlieb J, Mattner F, Weissbrodt H, et al. Impact of graft colonization with gram-negative bacteria after lung transplantation on the development of bronchiolitis obliterans syndrome in recipients with cystic fibrosis. Respir Med 2009;103:743-9.

102. Vos R, Vanaudenaerde BM, De Vleeschauwer SI, Van Raemdonck DE, Dupont LJ, Verleden GM. De novo or persistent pseudomonal airway colonization after lung transplantation: importance for bronchiolitis obliterans syndrome? Transplantation 2008;86:624-5; author reply 35-6.

103. Botha P, Archer L, Anderson RL, et al. Pseudomonas aeruginosa colonization of the allograft after lung transplantation and the risk of bronchiolitis obliterans syndrome. Transplantation 2008;85:771-4.

104. Vital D, Hofer M, Benden C, Holzmann D, Boehler A. Impact of sinus surgery on pseudomonal airway colonization, bronchiolitis obliterans syndrome and survival in cystic fibrosis lung transplant recipients. Respiration 2013;86:25-31.

105. Tipton L, Ghedin E, Morris A. The lung mycobiome in the next-generation sequencing era. Virulence 2017;8:334-41.

106. Delhaes L, Monchy S, Frealle E, et al. The airway microbiota in cystic fibrosis: a complex fungal and bacterial community--implications for therapeutic management. PLoS One 2012;7:e36313.

107. Harrison M, Twomey K, Mccarthy Y, et al. The Role Of Second-generation Sequencing To Characterize The Fungal Microbiota In The Adult Cystic Fibrosis Airway, And Its Correlation With Standard Culture-based Methods And Clinical Phenotype. Pediatric Pulmonology 2012;47:322. 108. Willger SD, Grim SL, Dolben EL, et al. Characterization and quantification of the fungal microbiome in serial samples from individuals with cystic fibrosis. Microbiome 2014;2:40. 
109. Kim SH, Clark ST, Surendra A, et al. Global Analysis of the Fungal Microbiome in Cystic Fibrosis Patients Reveals Loss of Function of the Transcriptional Repressor Nrg1 as a Mechanism of Pathogen Adaptation. PLoS Pathog 2015;11:e1005308.

110. Bafadhel M, McKenna S, Agbetile J, et al. Aspergillus fumigatus during stable state and exacerbations of COPD. Eur Respir J 2014;43:64-71.

111. Cui L, Lucht L, Tipton L, et al. Topographic diversity of the respiratory tract mycobiome and alteration in HIV and lung disease. Am J Respir Crit Care Med 2015;191:932-42.

112. Weigt SS, Elashoff RM, Huang $C$, et al. Aspergillus colonization of the lung allograft is a risk factor for bronchiolitis obliterans syndrome. Am J Transplant 2009;9:1903-11.

113. Willner $D$, Furlan $M$, Haynes $M$, et al. Metagenomic analysis of respiratory tract DNA viral communities in cystic fibrosis and non-cystic fibrosis individuals. PLoS One 2009;4:e7370.

114. Lysholm F, Wetterbom A, Lindau C, et al. Characterization of the viral microbiome in patients with severe lower respiratory tract infections, using metagenomic sequencing. PLoS One 2012;7:e30875.

115. Yang J, Yang F, Ren L, et al. Unbiased parallel detection of viral pathogens in clinical samples by use of a metagenomic approach. J Clin Microbiol 2011;49:3463-9.

116. Wylie KM, Mihindukulasuriya KA, Sodergren E, Weinstock GM, Storch GA. Sequence analysis of the human virome in febrile and afebrile children. PLoS One 2012;7:e27735.

117. Young JC, Chehoud C, Bittinger K, et al. Viral metagenomics reveal blooms of anelloviruses in the respiratory tract of lung transplant recipients. Am J Transplant 2015;15:200-9.

118. Abbas AA, Diamond JM, Chehoud C, et al. The Perioperative Lung Transplant Virome: Torque Teno Viruses are Elevated in Donor Lungs and Show Divergent Dynamics In Primary Graft Dysfunction. Am J Transplant 2016.

119. Garantziotis S, Howell DN, McAdams HP, Davis RD, Henshaw NG, Palmer SM. Influenza pneumonia in lung transplant recipients: clinical features and association with bronchiolitis obliterans syndrome. Chest 2001;119:1277-80.

120. Gottlieb J, Schulz TF, Welte T, et al. Community-acquired respiratory viral infections in lung transplant recipients: a single season cohort study. Transplantation 2009;87:1530-7.

121. Khalifah AP, Hachem RR, Chakinala MM, et al. Respiratory viral infections are a distinct risk for bronchiolitis obliterans syndrome and death. Am J Respir Crit Care Med 2004;170:181-7.

122. Kumar D, Erdman D, Keshavjee $S$, et al. Clinical impact of community-acquired respiratory viruses on bronchiolitis obliterans after lung transplant. Am J Transplant 2005;5:2031-6.

123. Costa C, Delsedime L, Solidoro P, et al. Herpesviruses detection by quantitative real-time polymerase chain reaction in bronchoalveolar lavage and transbronchial biopsy in lung transplant: viral infections and histopathological correlation. Transplant Proc 2010;42:1270-4.

124. Bakker NA, Verschuuren EA, Erasmus ME, et al. Epstein-Barr virus-DNA load monitoring late after lung transplantation: a surrogate marker of the degree of immunosuppression and a safe guide to reduce immunosuppression. Transplantation 2007;83:433-8.

125. Cao S, Strong MJ, Wang X, et al. High-throughput RNA sequencing-based virome analysis of 50 lymphoma cell lines from the Cancer Cell Line Encyclopedia project. J Virol 2015;89:713-29.

126. Engelmann I, Welte T, Fuhner T, et al. Detection of Epstein-Barr virus DNA in peripheral blood is associated with the development of bronchiolitis obliterans syndrome after lung transplantation. J Clin Virol 2009;45:47-53.

127. Finlen Copeland CA, Davis WA, Snyder LD, et al. Long-term efficacy and safety of 12 months of valganciclovir prophylaxis compared with 3 months after lung transplantation: a single-center, long-term follow-up analysis from a randomized, controlled cytomegalovirus prevention trial. J Heart Lung Transplant 2011;30:990-6.

128. Iwasenko JM, Scott GM, Naing Z, Glanville AR, Rawlinson WD. Diversity of antiviral-resistant human cytomegalovirus in heart and lung transplant recipients. Transpl Infect Dis 2011;13:145-53. 
129. Kerschner H, Jaksch P, Zweytick B, Puchhammer-Stockl E. Detection of human cytomegalovirus in bronchoalveolar lavage fluid of lung transplant recipients reflects local virus replication and not contamination from the throat. J Clin Microbiol 2010;48:4273-4.

130. Hammond SP, Martin ST, Roberts K, et al. Cytomegalovirus disease in lung transplantation: impact of recipient seropositivity and duration of antiviral prophylaxis. Transpl Infect Dis 2013;15:163-70.

\section{Figures:}

Figure 1:

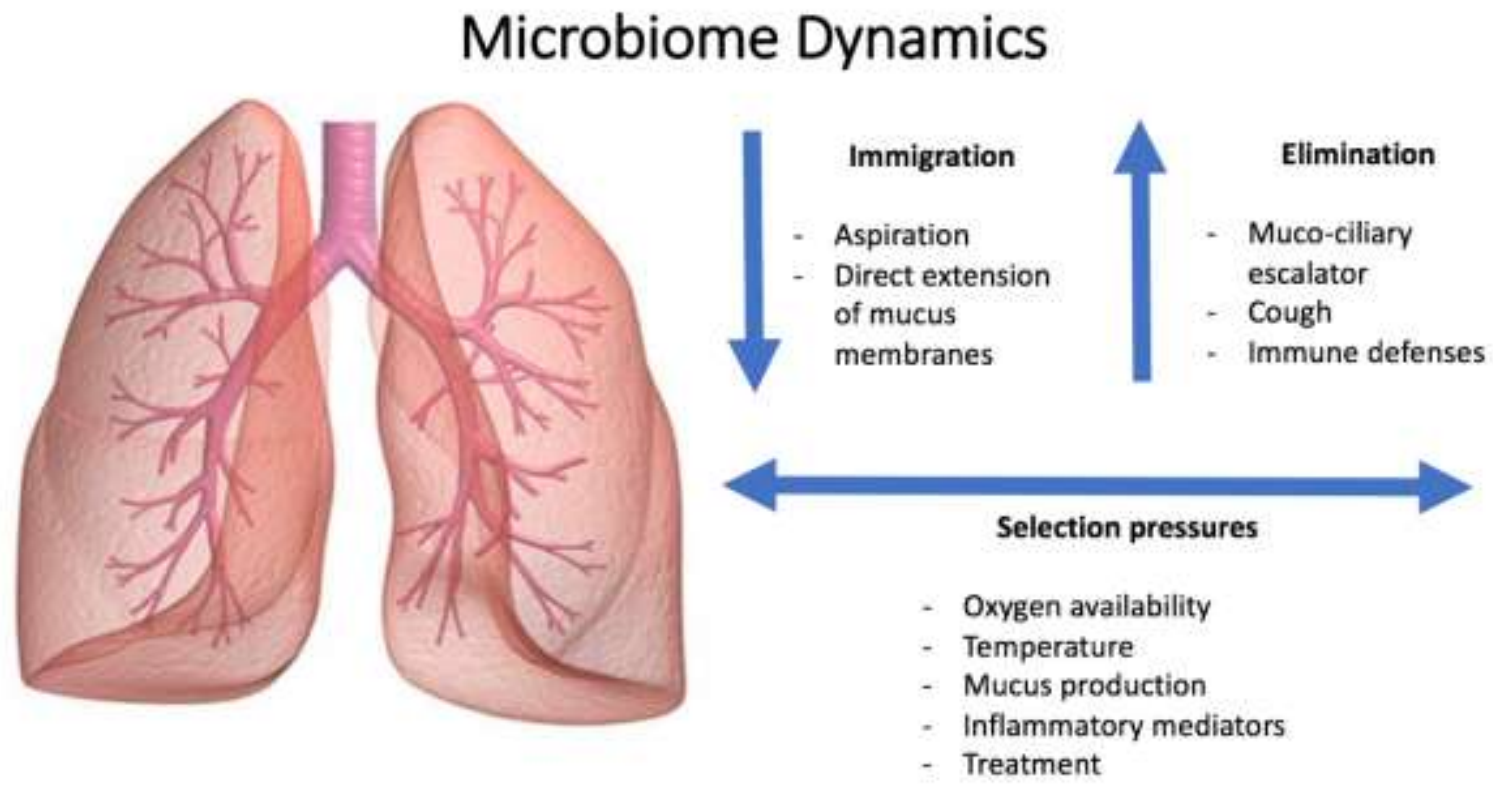

Figure 2:

\section{Dysbiosis of the Lung Microbiome after Lung Transplantation}

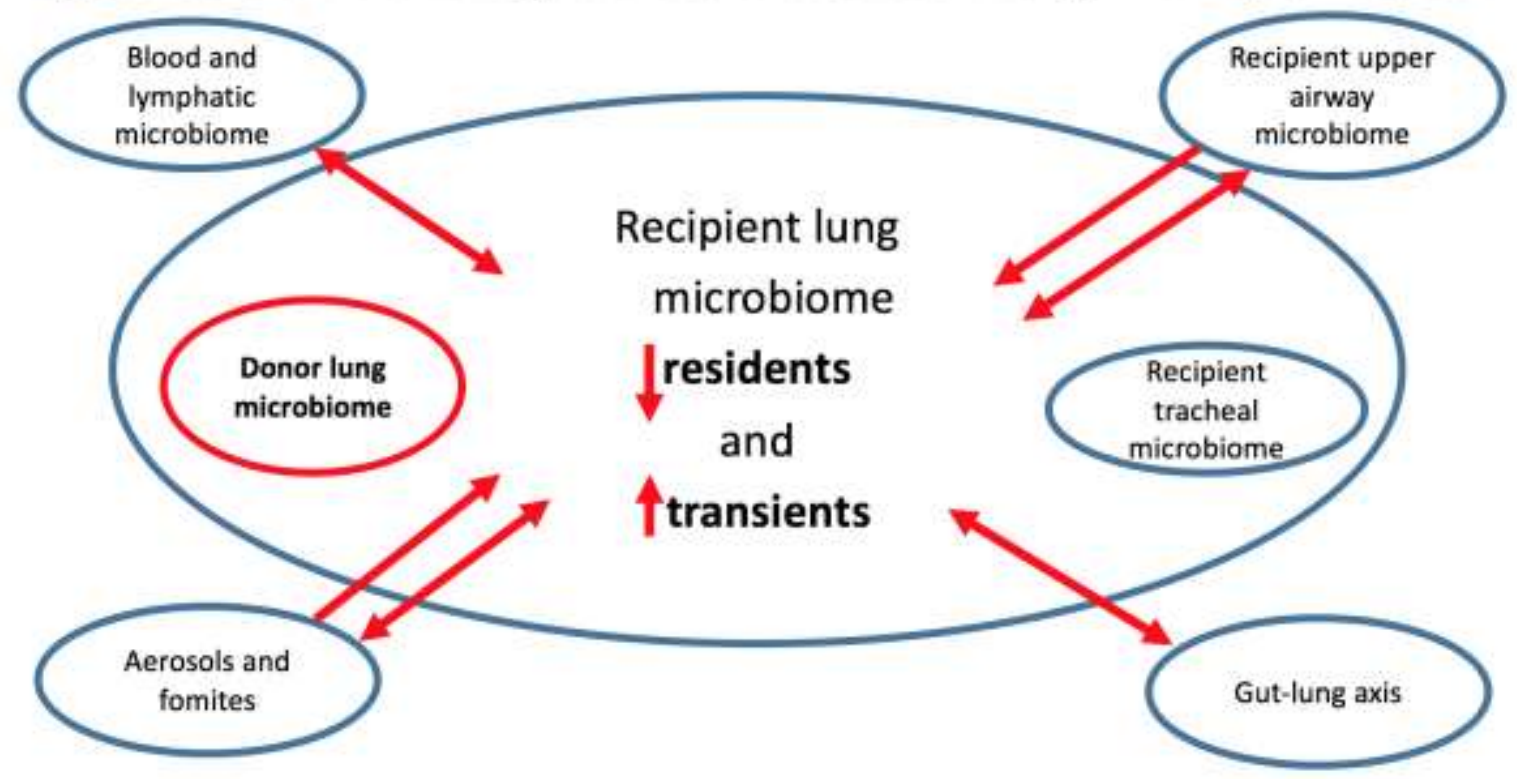


Figure 3:

\section{Dynamics of the Human Respiratory Virome after Lung Transplantation}

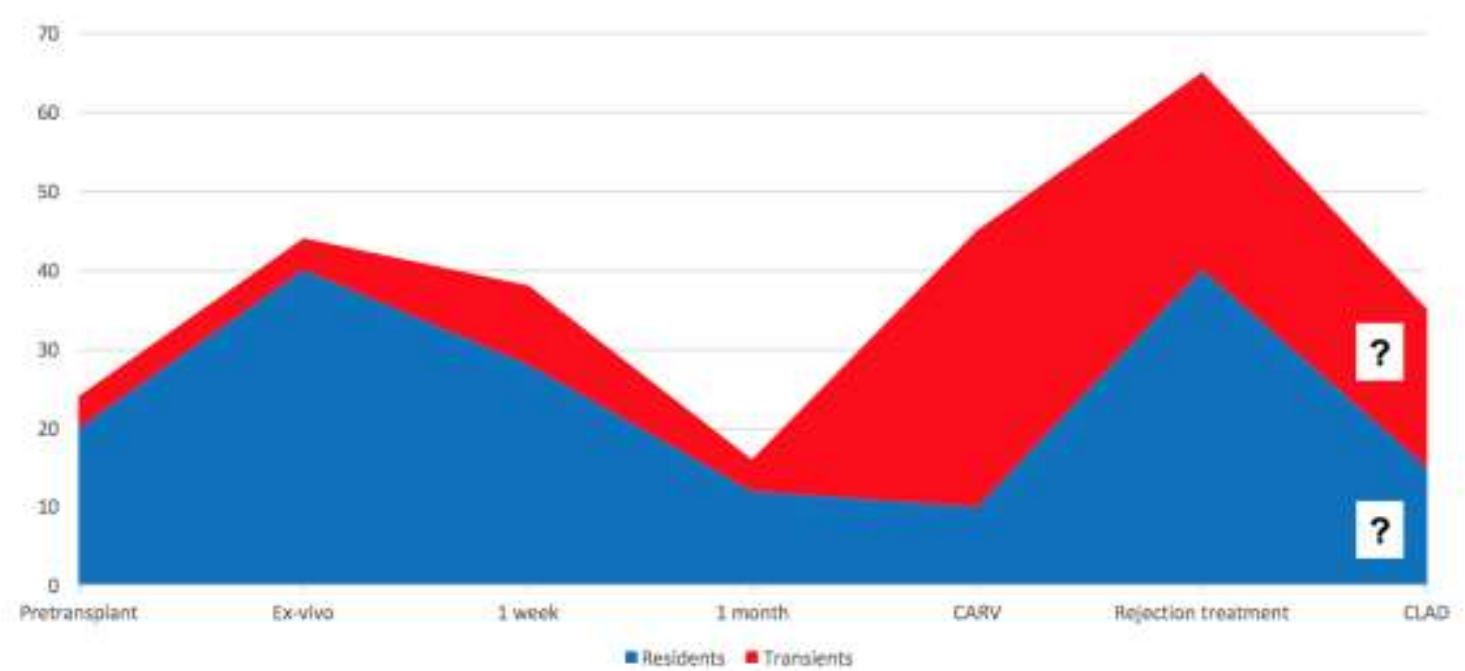

Figure Legends:

\section{Figure 1: Dynamics of the Human Respiratory Microbiome}

The human respiratory microbiome comprises all organisms that live in or on the human lung and respiratory tract including bacteria, fungi, archaea, viruses and bacteriophages. Numerous forces combine to create a dynamic situation such that the net result represents the balance between acquisition, elimination and local defense measures designed to maintain an equilibrium between resident and transient species.

\section{Figure 2: Dysbiosis of the Lung Microbiome after Lung Transplantation}

After lung transplantation, the healthy lung equilibrium between resident and transient species may be challenged by the donor microbiome transplanted within the new lungs which may be qualitatively and quantitatively different from the microbiome of the explanted native lungs. Other external events can also lead to dysbiosis including community acquired respiratory virus infection, the ex-vivo stage of lung procurement and the impact of immune suppression.

Figure 3: Dynamics of the Human Respiratory Virome after Lung Transplantation 
This concept diagram demonstrates a potential temporal relationship between resident viral species within the lung and transient species. Specific events lead to blooms of resident species such as the ex-vivo stage of lung procurement and heightened immune suppression. It is likely but not proven that acute community acquired respiratory virus (CARV) infection may suppress resident species temporarily while the impact of the development of chronic lung allograft dysfunction (CLAD) may depend on phenotype and also on therapies employed. 